\title{
Estructura del Test de Dependencia a los Videojuegos, relación con el juego, diferencias sexuales y tipologías de dependencia al juego en una muestra colombiana
}

\author{
Anthony Millán ${ }^{1}$, Moisés Mebarak ${ }^{1}$, Martha Martinez-Banfi ${ }^{2}$, Jeyleen Blanco ${ }^{1}$, Daniela Rodríguez ${ }^{1}$, \\ María D’Aubeterre ${ }^{3}$ y Mariano Chóliz ${ }^{4}$ \\ ${ }^{1}$ Universidad del Norte, Barranquilla, Colombia \\ ${ }^{2}$ Universidad Simón Bolivar, Barranquilla, Colombia \\ ${ }^{3}$ Fundación Universitaria Ceipa Business School, Barranquilla, Colombia \\ ${ }^{4}$ Universidad de Valencia, Valencia, España
}

\begin{abstract}
Structure of the Video Game Dependence Test, relationship with playing, sex differences and typology of gambling dependence in a Colombian sample
\end{abstract}

\begin{abstract}
The objective of this study was to analyze the factorial structure and psychometric properties of the Video Game Dependence Test (TDV; Cholíz \& Marco, 2011) in a Colombian sample, its ability to predict weekly game time, as well as differences by sex. The TDV was completed by a sample of 738 participants aged from 16 to 38 years. The factorial structure of the TDV was composed of these factors: impulsive gambling, compulsive gambling, and associated problems, constituting excellent predictors of weekly gambling time. Finally, we found 4 types of Video Game Dependence (DV): occasional gamer without DV, Gamer with DV awareness, Problematic gamer without DV awareness, and Frequent gamer without DV, 2 of which have a problematic relationship with video games.
\end{abstract}

Keywords: Problematic gambling; dependence on videogames; psychological addiction; gamers; play frequency.

Resumen: El objetivo del presente estudio fue examinar la estructura factorial y las propiedades psicométricas del Test de Dependencia de Videojuegos (TDV; Cholíz y Marco, 2011) con población colombiana, su capacidad para predecir el tiempo de juego semanal, así como las diferencias en función del sexo. El TDV fue cumplimentado por una muestra de 738 participantes, con edades entre los 16 y los 38 años. La estructura factorial de la TDV estaba compuesta por los siguientes factores: juego impulsivo, juego compulsivo y percepción de problemas asociados, los cuales fueron excelentes predictores del tiempo de juego semanal. Finalmente, encontramos 4 tipos de dependencia a los videojuegos (DV): jugador ocasional sin DV, jugador con conciencia de DV, jugador problemático sin conciencia de DV y jugador frecuente sin DV, de los cuales 2 de ellos tienen una relación problemática con los videojuegos.

Palabras clave: Juego problemático; dependencia a los videojuegos; adicción psicológica; video jugadores; frecuencia de juego.

Recibido: 04 de julio 2020; aceptado: 12 de febrero 2021

Correspondencia: Anthony Constant Millán de Lange, Departamento de Psicología, Universidad del Norte, Edificio E. Oficina 3-15E, Km 5, vía Puerto Colombia, 080001 Barranquilla, Colombia. Correo-e: delangea@uninorte.edu.co

\section{Introducción}

Actualmente no existe un consenso entre la $11^{\mathrm{a}}$ versión del manual de Clasificación Internacional de Enfermedades (CIE-11) de la Organización Mundial de la Salud (OMS) publicada en el 2019 y la $5^{\text {a }}$ versión del Manual Diagnóstico y Estadístico de los Trastornos 
Mentales (DSM-5) de la Asociación de Psiquiatría Americana (APA), publicada en el 2013, sobre el abordaje del uso excesivo de los videojuegos. Por un lado, la CIE-11 (OMS, 2019) lo considera una adicción, mientras que el DSM-5 (APA, 2013) lo propone para estudios futuros. No obstante, ambos manuales coinciden en que el juego excesivo es un problema en aumento (Mentzoni et al., 2011) que prevalece en jóvenes, hombres y/o personas que tienen pobre salud psicosomática, entre otros factores (Wittek et al., 2016).

La CIE-11 (OMS, 2019) considera que el trastorno por uso de videojuegos (6C51) no se restringe a una plataforma de juego (entiéndase plataforma de juego: la computadora personal, el celular, la tableta, la consola o cualquier dispositivo utilizado para acceder al juego, ya sea que el mismo esté en línea por Internet o no, p.ej., cartuchos, chips, CD-ROM, etc.), ya que en este trastorno se agrupan tanto a aquellos patrones de comportamiento de juego persistente o recurrente que se ejecutan: predominantemente en línea (6C51.0), predominantemente fuera de línea (6C51.1) y sin especificación (6C51.Z). El 6C51 se diagnostica de acuerdo con el CIE-11 (OMS, 2019), a partir de los siguientes criterios: falta de control en cuanto al inicio, aumento de la importancia que se otorga al juego e incremento del consumo del juego a pesar de las consecuencias.

Por otro lado, el DSM-5 (APA, 2013) incluye al «trastorno de juego por internet» (APA, 2013, p. 795), dentro de su sección 3 de «afecciones que necesitan más estudio» (APA, 2013, p. 783); ello es así porque se consideró que la evidencia era insuficiente como para justificar su inclusión «en la Sección II como diagnósticos oficiales de los trastornos mentales [y se] confía en que la investigación en este ámbito permitirá comprender mejor estas afecciones y que proporcionará información de cara a tomar decisiones acerca de su posible inclusión en las subsiguientes versiones del DSM» (APA, 2013, p.783). Los criterios diagnósticos de la DSM-5 (APA, 2013) para el trastorno de juego por Internet son: preocupación, tolerancia, retirada, persistencia, escape, problemas, decepción, desplazamiento y conflicto, los cuales no solo aplican para los videojuegos que están en Internet, sino que «también podrían estar implicados juegos de ordenador que no sean por Internet, aunque se ha investigado menos acerca de estos últimos» (APA, 2013, p. 796). Las diferencias entre las definiciones y los criterios diagnósticos del 6C51 del CIE-11 (OMS, 2019) y del trastorno de juego por Internet del DSM-5 (APA, 2013), ha llevado a críticas sobre la validez de los mismos (Király et al., 2017), así como también se ha criticado al proceso metodológico utilizado para validar los instrumentos de medición de esta condición o trastorno
(Aarseth et al., 2016). Dejando clara la necesidad de más y mejor evidencia empírica (Pontes y Griffiths, 2014) que permita identificar las características de la dependencia a los videojuegos (DV), así como el desarrollo de instrumentos de evaluación que permitan medirlo de forma válida (Király et al., 2017; Marengo et al., 2015), pues muchos de ellos no han sido validados de manera adecuada y presentan criterios inconsistentes (King et al., 2013; Király et al., 2017).

Con base en lo anterior, algunos autores (Aarseth et al., 2016, Ko et al., 2014, Müller, 2017, Van Rooij et al., 2017) consideran que la investigación psicométrica sobre la adicción a los videojuegos (AV) cobra especial relevancia dada la posibilidad de falsos positivos en el diagnóstico clínico, más aún cuando muchos de ellos se hacen por medio de cuestionarios de autoinforme (Király et al., 2017) y, por ello, destacan la importancia de la predicción como una fuente de validez externa. Una manera de evitar lo anterior es utilizando normas de corrección basadas en métodos refinados, es decir, estimadas por los métodos de regresión, Bartlett o Anderson-Rubin (DiStefano et al., 2009) dado que no poseen error de medida con respecto al puntaje factorial, al calcularse con base en el peso relativo que representa cada ítem del test en cada factor, de forma estandarizada. En cualquiera de los casos, por ser la adición a los videojuegos o el juego problemático una categoría diagnóstica reciente, se requiere que los instrumentos que la midan posean numerosos estudios que verifiquen su validez y fiabilidad, de manera que se pueda reducir a futuro la posibilidad de falsos positivos en el diagnóstico.

Actualmente existen diversos instrumentos para medir la AV o el juego problemático (Chóliz y Marco, 2011; Lemmens et al., 2015; Tejeiro y Bersabé, 2002; Van Rooij et al., 2012 y Young, 2015), sin embargo, el estudio de sus propiedades en Latinoamérica sólo ha tenido lugar con el instrumento de Pinzon y Briñez (2018), que ya ha sido estudiado en Colombia y que trata de una adaptación de la adicción a sustancias al contexto de la adicción a Internet y al videojuego y el Test de Dependencia a los Videojuegos (TDV) de Chóliz y Marco (2011) el cual es un instrumento específico del contexto de los videojuegos que cuenta con un estudio en Perú (Salas-Blas et al., 2017) siendo este último el instrumento que será utilizado en el presente estudio.

En el estudio de Chóliz y Marco (2011) el TDV no contó con la minimización de los sesgos de indeterminación factorial y confirmatorio, pues no se presentaron indicadores de la existencia de una estructura factorial subyacente, no se detallaron los criterios utilizados para identificar la existencia de la estructura de 4 factores de primer nivel y no se enumeraron, describieron y contras- 
taron los modelos factoriales alternativos. Este aspecto se reconoce posteriormente en la validación peruana (Salas-Blas et al., 2017) pero únicamente se agregan 2 modelos factoriales explicativos a los análisis, asumiendo la presencia de un puntaje total, ya sea: 1) de primer nivel $u$ orden o 2) de segundo nivel $u$ orden (se entiende como puntaje de segundo nivel u orden a aquel un nuevo puntaje factorial, que se vinculan a otros factores de primer nivel y que resume aún más la información original de los ítems, de lo que harían los factores de primer nivel que se vinculan a estos directamente), en la estructura pre-establecida de 4 factores. No obstante, Chóliz y Marco (2011) describen que el uso de un puntaje total del TDV tiene una excelente correlación $(r=.62)$ estadísticamente significativa $(p<.01)$ con la frecuencia semanal de juego (FSJ) como criterio de validación externa al TDV, «sin embargo, en los resultados reportados no se encontraron datos empíricos ni la racionalidad para crear un puntaje total» (Salas-Blas et al., 2017, p. 3).

Esta investigación pretende analizar la estructura factorial del TDV en adolescentes y jóvenes colombianos, para determinar si se mantiene el mismo número de factores y la misma organización de sus ítems a las propuestas por Chóliz y Marco (2011) y por Salas-Blas et al., (2017), a fin de proporcionar una mejor información sobre su validez interna, al minimizar tanto el sesgo de capitalización del azar como los sesgos confirmatorio y de indeterminación factorial, para luego presentar los parámetros de corrección bajo la metodología refinada de regresión, con el fin de asegurar una medida más precisa del constructo que representa. Se describe también la consistencia interna que aplica para modelos factoriales (coeficiente omega). Como fuentes de validación externa a la estructura interna del TDV, se verifica: si se mantienen las diferencias esperadas por sexo a favor de los hombres (Wittek et al., 2016) y si hay una adecuada predicción de la FSJ, considerando que aquellos «con un trastorno de juego por Internet grave dedicarán más horas al ordenador y tendrán una mayor pérdida de relaciones o de oportunidades laborales o escolares» (APA, 2013 p. 796), ya sea porque a mayor cantidad de tiempo de uso de video juegos, se llega incluso «a interferir con pautas de conducta higiénicas o saludables y hasta perturbar las relaciones familiares» (Choliz y Marco, 2011, p. 419), ya sea porque ella es un indicador para medir los efectos neurobiológicos de los vídeo juegos (Buiza-Aguado et al., 2017) y de hecho es también una medida de uso común en el contexto de adicciones en general (Walther et al., 2012). Finalmente, se presenta una tipología diagnóstica, que ayudará al profesional de la salud en la valoración del caso y la planificación de su intervención clínica.

\section{Método}

\section{Participantes}

Para este estudio la muestra fue de 738 participantes, superando los criterios de: 1) representatividad, estimados a partir de la población proyectada para el año 2017 por el Departamento Administrativo Nacional de Estadística de Colombia (DANE, 2018) que indica que el mínimo de participantes debería ser 722 personas; 2) la potencia estadística para el análisis factorial exploratorio (AFE) y/o para el análisis factorial confirmatorio (AFC) (Hair et al., 2014), que supondría al menos 250 participantes para el TDV; y 3 ) el criterio que establece a una muestra grande a partir de $n=500$ ), en estudios de validez de constructo (Hernández et al., 2016; Prieto y Muñiz, 2000).

La muestra está compuesta de forma balanceada $\left(\chi^{2}=\right.$ $0.00 ; p=.98 ; w=0.01)$ por sexo, ya que el $50.68 \%$ eran mujeres $(2.44 \%$ eran menores de 18 años y el $48.24 \%$ eran mayores de 18 años) y el $49.32 \%$ eran hombres (1.49\% eran menores de 18 años y el $47.83 \%$ eran mayores de 18 años), con edades entre 16 y 38 años $(M=20.3$, $D T=2.27$ ). Se observó que dicha muestra no tuvo diferencias estadísticamente significativas por sexo $(Z=$ $0.047)$ ni por edad $(Z=0.00)$ con respecto a la población proyectada para el año 2017 por el DANE (2018). Para el análisis de la relación del TDV con la FSJ hubo una reducción del tamaño de la muestra ya que 23 participantes no contestaron esta pregunta; con lo cual la muestra pasó de 738 a 715 participantes, manteniendo su balanceo por sexo $\left(\chi^{2}=0.023 ; p=.87 ; w=0.01\right)$; ya que el $50.77 \%$ eran mujeres $(2.30 \%$ eran menores de 18 años y el $46.88 \%$ eran mayores de 18 años) y el restante $49.23 \%$ eran hombres $(1.49 \%$ eran menores de 18 años y el $46.21 \%$ eran mayores de 18 años), con edades entre 16 y 38 años $(M=20.3, D T=2.29)$. Al comparar las características de ambas muestras, no hubo diferencias estadísticamente significativas entre ellas por sexo $\left(\chi^{2}=.001 ; p\right.$ $=.97 ; w=0.021)$ o edad $(t=-0.128 ; p=.898 ; d=0.008)$, por lo que las conclusiones obtenidas en cada una de ellas son equiparables entre sí.

\section{Instrumentos}

Cuestionario sociodemográfico: Cuestionario de autoinforme en el que se preguntó: sexo (masculino, femenino), edad, ciudad de procedencia y nivel educativo (bachillerato, universitario incompleto, universitario completo, posgrado incompleto, posgrado completo). Por ser una encuesta social (Briones, 1996), que no implica la presencia de un constructo subyacente o de una 
escala sumativa, no supone la presencia de indicadores psicométricos.

Cuestionario de hábitos de uso de video juegos: Cuestionario de autoinforme donde se preguntó sobre: a) la frecuencia de juego semanal en días (a diario, 5-6 veces a la semana, 3-4 veces a la semana, 1-2 días a la semana, muy esporádicamente, no juego); b) la frecuencia de juego semanal en horas, tanto en los días de estudios y/o laborales como durante los fines de semanas y festivos; c) nombres de los videojuegos a los que se juega con mayor frecuencia; d) modalidad de juego (online $u$ offline); e) si se juega desde el celular (si, no); f) si se juega desde las redes sociales (sí, no) y g) si se juega a algún tipo de juego de azar online: apuestas deportivas, casinos o bingos online, póker, etc. (si, no). Por ser una encuesta social (Briones, 1996), que no implica la presencia de un constructo subyacente o de una escala sumativa, no supone la presencia de indicadores psicométricos.

Test de Dependencia a los Videojuegos (TDV; Chóliz y Marco, 2011): Se trata de una escala de autoinforme de 25 ítems que evalúan desde una perspectiva de prevención el grado en que el uso de videojuegos «pueden llegar a provocar un proceso de dependencia, que es la característica sustancial de la adicción» (Chóliz y Marco, 2011, p. 424). La escala se contesta según un rango de respuestas de cinco puntos, que varía entre 0 («completamente en desacuerdo») y 5 («completamente de acuerdo»). Se ha proporcionado evidencia sobre su alta consistencia interna en cada una de las validaciones que han sido realizadas ( $\alpha$ mayores a .94). En España, Chóliz y Marco (2011) describen cuatro componentes (abstinencia, abuso y tolerancia, problemas ocasionados por los videojuegos y dificultad en el control), mientras que, en Perú Salas-Blas et al. (2017), concluyen que el TDV posee una estructura unifactorial.

\section{Procedimiento}

La recolección de la muestra fue por un muestreo no probabilístico por conveniencia. Se realizó por medio de un formulario de Google entre julio de 2016 y febrero de 2017. Se contó con el apoyo de un equipo de voluntarios, estudiantes de Psicología de la Universidad del Norte, los cuales fueron entrenados para la aplicación de la prueba de manera online, quienes facilitaron el enlace de acceso a personas cercanas, compañeros, familiares, entre otros. Para el entrenamiento, se siguieron las especificaciones de Eiroa, Fernandez y Perez (2008) que señala los aspectos a considerar para el proceso de levantamiento de información en investigación psicológica en Internet, específicamente en lo que respecta al contacto con usuarios a partir de listas de correo, través de una Web (como serían las Redes Sociales), contacto con usuarios de foros y finalmente contacto con usuarios de chats. Todos los sujetos aceptaron participar de manera voluntaria y se garantizó que la información seria únicamente para fines de investigación, así mismo se resaltó a los participantes y a los voluntarios que apoyaron el libre abandono del estudio en cualquier fase de su desarrollo y el anonimato de la información recogida.

\section{Análisis estadístico}

De las dos metodologías actuales para la validación de estructuras factoriales en test psicológicos (Lloret et al., 2014), elegimos aquella que permite realizar un uso secuencial del AFE y AFC de forma que con la primera técnica se impongan restricciones mínimas que permitan obtener diferentes soluciones factoriales iniciales y que, posteriormente, le serán impuestas «restricciones mucho más fuertes que permiten poner a prueba una solución única, cuyo ajuste puede ser evaluado utilizando diferentes índices de bondad de ajuste» (Lloret et al., 2014, p. 1155). De esta forma, se minimiza el sesgo confirmatorio (Hair et al., 2014) y, con ello, el error de indeterminación factorial. Se aplicó el AFE, método de componentes principales y residuales mínimos (MINRES), según las matrices de correlación inter ítem utilizadas: Pearson $\left(\mathrm{MC}_{\text {Pearson }}\right)$ y policóricas $\left(\mathrm{MC}_{\text {Policóricas }}\right)$, respectivamente. En ambos casos, se utilizaron rotaciones varimax o promax, dado que la primera es la que cumple con el supuesto de independencia factorial necesario para la predicción de la FSJ, mientras que la segunda fue la utilizada por Chóliz y Marco (2011) y Salas-Blas et al. (2017). Para identificar el mayor número de modelos factoriales en el AFE, se utilizaron los criterios clásicos (Hair et al., 2014) de: autovalor mayor a $1(\lambda>1)$, porcentaje de varianza explicada acumulada $\left(\% \sigma_{\text {exp }}^{2}\right)$ superior al $60 \%\left(\% \sigma_{e x p}^{2},>60 \%\right)$ y contraste de caída. Como criterios a priori, se utilizaron los modelos descritos por Chóliz y Marco (2011) o por Salas-Blas et al. (2017). Adicionalmente, se utilizaron los criterios basados en re-muestreo (Lorenzo-Seva y Ferrando, 2013) a saber: análisis paralelo clásico (CPA), el análisis paralelo óptimo (OPA) y el de promedio parcial mínimo (MAP).

Para determinar cuál de los modelos anteriores tenía el mejor ajuste con las respuestas al TDV se utilizaron los indicadores de ajuste: absoluto, incremental y parsimonia, calculados en el AFC, utilizando los métodos de máxima verosimilitud (ML) y mínimos cuadrados no ponderados (ULS). Para el análisis de consistencia interna se utilizó el coeficiente (McDonald, 1999), siendo el más adecuado para el contexto de test factoriales (Dunn 
et al., 2013). Una vez obtenido(s) el o los modelo(s) con mejor(es) ajuste(s), se presentaron las normas de corrección e interpretación del TDV y se analizó su relación con la FSJ en horas. Para determinar las diferencias por sexo, se calculó el estadístico $t$ de Student, así como su significancia ( $p$ ) y tamaño del efecto ( $d$ de Cohen). Para determinar la capacidad predictiva del TDV con respecto a la FSJ, se analizó el coeficiente $r$ o $R$ (regresión simple o múltiple, respectivamente) dependiendo de si el modelo ganador fuese unifactorial o multifactorial. En el caso del cálculo de $R$, se verificó que previamente se cumpliera con el supuesto de ausencia de colinealidad a través del indicador de tolerancia y del factor de inflación de la varianza (VIF). Para establecer la tipología de video jugador problemático se realizó un análisis de clúster con los criterios de agrupación bayesiano o de Schwarz y de información de Akaike y, posteriormente, se llevó a cabo una comparación estadística con el análisis de varianza (ANOVA) o la $t$, dependiendo de si la tipología era mayor a 2 grupos o de mínimo 2 grupos, de esta forma se validó de forma externa cuál sería el o lo(s) clúster(s) que consideraríamos problemático(s) al tener una mayor presencia de FSJ. Finalmente, se determinó si en de cada tipología existía una proporción más allá del azar de hombres o mujeres, a través del $\chi^{2}$ así como su $p$ y tamaño del efecto ( $d$ Cohen).

\section{Resultados}

Existe una estructura factorial al obtener un valor determinante cercano a .00 tanto para la $\mathrm{MC}_{\text {pearson }}\left(1.81 \times 10^{-}\right.$ ${ }^{8}$ ) como para la $\mathrm{MC}_{\text {policórica }}\left(1.20 \times 10^{-12}\right)$. La adecuación muestral de Kaiser, Meyer y Olkin (KMO) fue mayor al criterio de $0.50\left(\mathrm{MC}_{\text {pearson }}=0.96 ; \mathrm{MC}_{\text {policórica }}=0.95\right)$. La significancia de la medida de esfericidad de Bartlett fue menor a $.05\left(\mathrm{MC}_{\text {pearson }}=.00 ; \mathrm{MC}_{\text {policórica }}=.00\right)$.

Se determinó la existencia de 9 modelos factoriales posibles, cuya descripción se encuentra en la Tabla 1. El modelo 5.1, no se obtuvo de los cálculos del AFE, sino que proviene del estudio de Chóliz y Marco (2011). Los modelos $1.2,2.2,4.2$ y 5.2 son similares a los modelos $1.1,1.2,4.1$ y 5.1 , con la diferencia de habérseles agregado un puntaje total de segundo nivel u orden, siguiendo las recomendaciones de Salas-Blas et al. (2017). De estos, se rechazaron los modelos 4.1 y 4.2 , debido a que los mismos se configurarían en el AFC como un caso Heywood (Kolenikov y Bollen, 2012), al tener un único ítem dentro de un factor (el Factor 1), lo cual es una solución impropia (Freiberg, Stover, De la Iglesia, y Fernandez, 2013) o una especificación incorrecta (Kolenikov y Bollen, 2012).

Tabla 1. Organización de los ítems de acuerdo a los factores de cada uno de los modelos

\begin{tabular}{|c|c|c|c|c|c|c|}
\hline \multirow{2}{*}{ Modelos } & \multicolumn{2}{|c|}{ Niveles de los factores } & \multicolumn{4}{|c|}{ Distribución de ítems por factor } \\
\hline & Nivel I & Nivel II & Factor 1 & Factor 2 & Factor 3 & Factor 4 \\
\hline 1.1 & 3 & 0 & 1 al 6 y del 8 al 14 & $7,15,16,18,19,20,21$ & $17,22,23$ al 25 & N/A \\
\hline 1.2 & 3 & 1 & & & & \\
\hline 2.1 & 2 & 0 & 1 al 14 & 15 al 25 & $\mathrm{~N} / \mathrm{A}$ & $\mathrm{N} / \mathrm{A}$ \\
\hline 2.2 & 2 & 1 & & & & \\
\hline 3.1 & 1 & 0 & 1 al 25 & N/A & N/A & N/A \\
\hline 4.1 & 3 & 0 & 1 & 2 al 14 & 15 al 25 & N/A \\
\hline 4.2 & 3 & 1 & 1 & 2 al 14 & 15 al 25 & N/A \\
\hline 5.1 & 4 & 0 & $3,4,6,7,10,11,13$ & $1,5,8,9$ y 12 & 16,17 & $2,15,18,20$ y 22 \\
\hline 5.2 & 4 & 1 & & & 19 у 23 & \\
\hline
\end{tabular}

Todo lo anterior supone que, para la segunda fase de análisis con los AFC, se probaron un total de 7 modelos factoriales, los cuales fueron estimados con los métodos de ML y ULS con la intención de minimizar el sesgo confirmatorio; si bien la kurtosis relativa multivariante (RMK) sugiere la presencia de una distribución normal multivariante de los errores $(\mathrm{RMK}=1.758)$. El resultado de los AFC supuso que el modelo 2.2, quedase descarta- do al no poder converger la solución factorial porque su matriz no estaba definida positivamente, ya que la varianza de error $\left(S_{E}^{2}\right)$ del Factor 2 era negativa $\left(S_{E \text {.Factor } 2}^{2}=\right.$ -0.27). Los resultados del resto de los modelos factoriales analizados, se encuentran descritos en las Tablas 2 y 3, según fuesen estimados por ML o ULS. 
Tabla 2. Medidas de ajuste absoluto para los factores evaluados

\begin{tabular}{cccccccccc}
\hline \multirow{2}{*}{ N. ${ }^{\circ}$ Modelo } & \multirow{2}{*}{ Método de estimación } & \multicolumn{7}{c}{ Medidas de ajuste absoluto } \\
\cline { 3 - 10 } & \multicolumn{2}{c}{$g l$} & $\chi^{2}$ & $\chi^{2} g l$ & GFI & ECVI & NCP & RMSR & RMSEA \\
\hline 1.1 & ULS & 272 & $2080.04^{*}$ & 7.65 & .99 & 1808.04 & 2.97 & .10 & $.095^{*}$ \\
1.1 & ML & 272 & $1961.61^{*}$ & 7.21 & .82 & 1689.61 & 2.81 & .09 & $.092^{*}$ \\
1.2 & ULS & 272 & $2080.04^{*}$ & 7.65 & .99 & 1808.04 & 2.97 & .10 & $.095^{*}$ \\
1.2 & ML & 272 & $1961.61^{*}$ & 7.21 & .82 & 1689.61 & 2.81 & .09 & $.092^{*}$ \\
2.1 & ULS & 274 & $2311.84^{*}$ & 8.44 & .99 & 2037.84 & 3.28 & .10 & $.100^{*}$ \\
2.1 & ML & 274 & $2318.10^{*}$ & 8.46 & .80 & 2044.10 & 3.28 & .10 & $.100^{*}$ \\
2.2 & ULS & 273 & $2311.79^{*}$ & 8.47 & .99 & 2038.79 & 3.28 & .10 & $.100^{*}$ \\
3.1 & ULS & 275 & $5581.39^{*}$ & 20.30 & .98 & 5306.39 & 7.71 & .16 & $.160^{*}$ \\
3.1 & ML & 275 & $5524.75^{*}$ & 20.09 & .63 & 5249.75 & 7.63 & .16 & $.160^{*}$ \\
\hline
\end{tabular}

Nota. Los 2 asteriscos $\left(^{* *}\right)$ señalan que el $p$ está entre .05 y .01, el asterisco simple $(*)$, señala que el $p$ es menor a .01. No se reportan la significación $(p)$ del RMSEA y del $\chi^{2}$ porque tienen el mismo valor de .00 en todos los modelos

A partir de las medidas de ajuste absoluto (Tabla 2), se observó que los modelos con mayor ajuste fueron: el $1.1_{\mathrm{ML}}$ y el $1.2_{\mathrm{ML}}$, siendo idénticas la estructura de primer nivel de ambos modelos, al estar compuestos por 3 Factores, y cuya única diferencia está en la presencia de un puntaje global de DV (modelo 1.2 $\mathrm{ML}$ ). Dicha conclusión se obtuvo al observar que ambos modelos presentaban los valores más bajos de Chi cuadrado $\left(\chi^{2}=1961.61\right)$, la razón chi-cuadrado/grado de libertad $\left(\chi^{2} / \mathrm{gl}=7.21\right)$, el índice de validación cruzada $(\mathrm{ECVI}=1689.61)$, índice de no centralidad $(\mathrm{NCP}$ $=2.81$ ), índice de residuos cuadráticos medio estandariza- dos $($ RMSR $=.09)$, además de tener el valor del error de aproximación cuadrático medio (RMSEA $=.092$ ), por debajo del límite de umbral superior (valor más cercano a los 0.10 puntos), al ser el tamaño de la muestra mayor a 100 participantes (Hair et al., 2014). Los valores de significación del RMSEA (RMSEA $)$ y del $\chi^{2}\left(\chi_{p}^{2}\right)$ no permiten distinguir los modelos entre sí, pues todos tienen el mismo valor de .00. En contraste con lo anterior, el índice de bondad de ajuste (GFI), sugiere como mejores modelos al: $1.1_{\mathrm{ULS}}, 1.2_{\mathrm{ULS}}, 2.1_{\mathrm{ULS}}$ y $2.2_{\mathrm{ULS}}$, por tener el indicador más alto entre los modelos comparados $(\mathrm{GFI}=.99)$.

Tabla 3. Medidas de ajuste incremental y parsimonia

\begin{tabular}{|c|c|c|c|c|c|c|c|}
\hline \multirow{2}{*}{ N. ${ }^{\circ}$ Modelo } & \multirow{2}{*}{ Método de estimación } & \multicolumn{4}{|c|}{ Medidas de ajuste incremental } & \multicolumn{2}{|c|}{ Medidas de ajuste de parsimonia } \\
\hline & & AGFI & NNFI o TLI & CFI & NFI & PNFI & PGFI \\
\hline 1.1 & ULS & .99 & 1.01 & 1.00 & 1.00 & $.91 * *$ & $.83^{*}$ \\
\hline 1.1 & ML & .79 & .97 & .97 & .96 & $.87^{*}$ & .69 \\
\hline 1.2 & ULS & .99 & 1.01 & 1.00 & 1.00 & $.91 * *$ & $.83^{*}$ \\
\hline 1.2 & ML & .79 & .97 & .97 & .96 & $.87^{*}$ & .69 \\
\hline 2.1 & ULS & .99 & 1.01 & 1.00 & 1.00 & $.91 * *$ & $.84 * *$ \\
\hline 2.1 & ML & .76 & .96 & .97 & .96 & $.88^{*}$ & .67 \\
\hline 2.2 & ULS & .99 & 1.01 & 1.00 & 1.00 & $.91 * *$ & $.83^{*}$ \\
\hline 3.1 & ULS & .97 & 1.01 & 1.00 & 1.00 & $.92 * *$ & $.83^{*}$ \\
\hline 3.1 & ML & .56 & .94 & .94 & .94 & $.86^{*}$ & .53 \\
\hline
\end{tabular}

Nota. Los 2 asteriscos (**) señalan el valor máximo de PNFI y PGFI, el asterisco simple (*), señala el valor que no tiene diferencia sustancial con el máximo, al no superar la diferencia de .09 puntos, siguiendo las recomendaciones de Hair et al. (2014).

Con respecto a los indicadores de ajuste incremental (ver Tabla 3), se llega a la misma conclusión de un mejor ajuste de los modelos $1.1_{\mathrm{ML}}$ y el $1.2_{\mathrm{ML}}$ a las respuestas del TDV, ya que el índice de bondad de ajuste absoluto no normado $(\mathrm{NNFI}=1.01)$, también llamado índice TukerLewis (TLI), son los más altos y se encuentran tanto por encima del criterio de .90 puntos, como del límite (NNFI $=1.00$ ) de lo que se esperaría cuando hay una parametri- 
zación del modelo (Batista y Coenders, 2000); lo mismo sucede con el índice de ajuste normado $(\mathrm{NFI}=1.00)$ y el índice de ajuste comparado (CFI $=1.00)$, quienes utilizan los mismos criterios de interpretación.

No obstante, y siguiendo los mismos criterios de comparación, en el índice ajustado de bondad de ajuste $($ AGFI $=0.99)$, se consideran los mejores modelos: $1.1_{\mathrm{ULS}}, 1.2_{\mathrm{ULS}}, 2.1_{\mathrm{ULS}}$ y $2.3_{\mathrm{ULS}}$. De acuerdo las medidas de ajuste de parsimonia, todos los modelos poseen un valor aceptable, al no tener una diferencia mayor al rango de .06 a .09 puntos con el valor más alto del Ajuste Normado de Parsimonia (PNFI $=0.92)$ y del índice de calidad de ajuste de parsimonia (PGFI $=0.84)$. En conclusión, los modelos $1.1_{\mathrm{ML}}$ y $1.2_{\mathrm{ML}}$, son los factores que mejor explican las respuestas del TDV y se caracterizan por tener 3 factores de primer nivel, aunque el modelo 1.2 presenta un factor de segundo nivel, independientemente de que se cumpla o no con el supuesto de normalidad.
Pese lo anterior, el valor de la RMK podría explicar el por qué los modelos factoriales que obtuvieron el mayor número de indicadores de ajuste, provenían de cálculos realizados a partir de la $\mathrm{MC}_{\text {Pearson }} \mathrm{y}$ con una estimación basada en ML, aun cuando las respuestas al TDV eran de naturaleza ordinal.

En este mismo orden de ideas, el modelo $1.1_{\mathrm{ML}}$ podría considerarse más parsimonioso que el modelo $1.2_{\mathrm{ML}}$, pues si bien ambos tienen el mismo $\% \sigma_{\text {exp }}^{2}\left(\% \sigma_{\text {exp }}^{2}=63.39 \%\right)$, siendo a su vez el más alto y sistemático $\left(\chi^{2}=7.177\right)$ entre todos los comparados, el modelo $1.1_{\mathrm{ML}}$ lo hace con el menor número de componentes (3 factores de primer nivel vs., 3 factores de primer nivel y 1 factor de segundo nivel). En ambos casos, la consistencia interna del modelo es excelente $(\Omega=0.99)$, si se interpreta a partir de los criterios descritos por Prieto y Muñiz (2000) o Hernández et al. (2016).

Tabla 4 Parámetros de corrección del TDV

\begin{tabular}{|c|c|c|c|c|c|}
\hline Indicador & $M$ & $D T$ & $\begin{array}{l}\text { Juego impulsivo } \\
\text { (Factor 1) }\end{array}$ & $\begin{array}{l}\text { Juego compulsivo } \\
\text { (Factor 2) }\end{array}$ & $\begin{array}{l}\text { Percepción de problemas asociados } \\
\text { (Factor 3) }\end{array}$ \\
\hline $\mathrm{p} 1$ & .94 & 1.26 & .06 & .12 & -.12 \\
\hline $\mathrm{p} 2$ & .37 & .86 & .21 & -.16 & -.01 \\
\hline p3 & .86 & 1.26 & .11 & .14 & -.19 \\
\hline $\mathrm{p} 4$ & .81 & 1.15 & .12 & .05 & -.09 \\
\hline $\mathrm{p} 5$ & .72 & 1.15 & .11 & -.02 & .00 \\
\hline p6 & .43 & .91 & .19 & -.21 & .10 \\
\hline p7 & .12 & 1.40 & .08 & .19 & -.22 \\
\hline $\mathrm{p} 8$ & .59 & 1.03 & .17 & -.07 & -.03 \\
\hline p9 & .55 & .99 & .15 & -.09 & .03 \\
\hline $\mathrm{p} 10$ & .86 & 1.22 & .12 & .02 & -.06 \\
\hline p11 & .71 & 1.12 & .14 & .01 & -.09 \\
\hline p12 & .72 & 1.13 & .08 & -.02 & .04 \\
\hline p13 & .81 & 1.19 & .09 & .05 & -.05 \\
\hline p14 & .73 & 1.20 & .09 & -.09 & .11 \\
\hline $\mathrm{p} 15$ & .71 & 1.04 & -.06 & .07 & .11 \\
\hline p16 & .13 & 1.32 & -.09 & .23 & -.04 \\
\hline p17 & .60 & 1.02 & -.08 & -.06 & .29 \\
\hline $\mathrm{p} 18$ & .15 & 1.36 & -.08 & .27 & -.09 \\
\hline p19 & .14 & 1.34 & -.09 & .24 & -.04 \\
\hline $\mathrm{p} 20$ & 1.00 & 1.16 & -.06 & .13 & .05 \\
\hline $\mathrm{p} 21$ & .13 & 1.36 & -.08 & .25 & -.08 \\
\hline $\mathrm{p} 22$ & .58 & .91 & -.04 & -.03 & .22 \\
\hline $\mathrm{p} 23$ & .40 & .88 & -.05 & -.18 & .39 \\
\hline $\mathrm{p} 24$ & .43 & .85 & -.04 & -.15 & .35 \\
\hline $\mathrm{p} 25$ & .81 & 1.14 & -.07 & .01 & .20 \\
\hline DV & .00 & .10 & .01 & .71 & -.70 \\
\hline
\end{tabular}


Por otro lado, al ser calculados las puntuaciones factoriales, a través de los parámetros descritos en la Tabla 4, se determinó que la distribución del TDV está estandarizada $(M=0.00 ; D T=1.00)$, para todos los factores. También, se puede señalar, que la forma de las distribuciones en ninguno de estos factores representa a una curva normal, dado a que el $p$ de la Prueba de Kolmogorov-Smirnov con corrección de Lilliefors fue menor al criterio de .05 puntos en todas ellas; $y$, de hecho, las mismas están coleadas hacia el extremo derecho de la curva (asimetría negativa).

De acuerdo con la distribución de los ítems en el primer nivel de la estructura factorial de los modelos $1.1 \mathrm{y}$ 1.2 (ver Tabla 5), se le asignó el nombre de «juego impulsivo» al factor 1 , «juego compulsivo» al factor 2 y «percepción de problemas asociados», al factor 3.

Tabla 6 Parámetros de interpretación del TDV

\begin{tabular}{clllcl}
\hline Bisagra & \multicolumn{1}{c}{ Nivel } & $\begin{array}{c}\text { Juego impulsivo } \\
\text { (Factor 1) }\end{array}$ & $\begin{array}{c}\text { Juego compulsivo } \\
\text { (Factor 2) }\end{array}$ & $\begin{array}{c}\text { Percepción de problemas } \\
\text { asociados (Factor 3) }\end{array}$ & $\begin{array}{c}\text { Dependencia a los videojuegos } \\
\text { (Factor general) }\end{array}$ \\
\hline 5 & Muy alto & $\mathrm{Z}_{\mathrm{F} 1} \geq 1.55$ & $\mathrm{Z}_{\mathrm{F} 2} \geq 3.23$ & $\mathrm{Z}_{\mathrm{F} 3} \geq 1.25$ & $\mathrm{Z}_{\mathrm{DV}} \geq 2.26$ \\
4 & Alto & $1.55>\mathrm{Z}_{\mathrm{F} 1} \geq .32$ & $3.23>\mathrm{Z}_{\mathrm{F} 2} \geq .68$ & $1.25>\mathrm{Z}_{\mathrm{F} 3} \geq .20$ & $2.26>\mathrm{Z}_{\mathrm{DV}} \geq .56$ \\
3 & Esperado & $.32<\mathrm{Z}_{\mathrm{F} 1}>-.50$ & $.68<\mathrm{Z}_{\mathrm{F} 2}>-1.03$ & $.20<\mathrm{Z}_{\mathrm{F} 3}>-.50$ & $.56<\mathrm{Z}_{\mathrm{DV}}>-.58$ \\
2 & Bajo & $-.50 \geq \mathrm{Z}_{\mathrm{F} 1}>-1.73$ & $-1.03 \geq \mathrm{Z}_{\mathrm{F} 2}>-3.58$ & $-.50 \geq \mathrm{Z}_{\mathrm{F} 3}>-1.56$ & $-.58 \geq \mathrm{Z}_{\mathrm{DV}}>-2.29$ \\
1 & Muy bajo & $\mathrm{Z}_{\mathrm{F} 1} \leq-1.73$ & $\mathrm{Z}_{\mathrm{F} 2} \leq-3.58$ & $\mathrm{Z}_{\mathrm{F} 3} \leq-1.56$ & $\mathrm{Z}_{\mathrm{DV}} \leq-2.29$ \\
\hline
\end{tabular}

Para la interpretación cualitativa de los factores del TDV, se utilizaron las 5 bisagras de Tukey (1976), cuyos parámetros están indicados en la Tabla 6 , estableciéndose como nivel muy bajo, a las puntuaciones que se encontraron en la Bisagra ${ }_{1}$, como nivel bajo a aquellos que estén en la Bisagra ${ }_{2}$, como nivel esperado a aquellos que estén en la Bisagra ${ }_{3}$, como nivel alto a aquellos que estén en la Bisagra ${ }_{4}$ y como nivel muy alto a aquellos que estén en la Bisagra ${ }_{5}$.

Tabla 7 Parámetros de interpretación del TDV

\begin{tabular}{cccccc}
\hline Bisagra & $\begin{array}{c}\text { Niveles de } \\
\text { interpretación }\end{array}$ & $\begin{array}{c}\text { Juego impulsivo } \\
\text { (Factor 1) }\end{array}$ & $\begin{array}{c}\text { Juego compulsivo } \\
\text { (Factor 2) }\end{array}$ & $\begin{array}{c}\text { Percepción de proble- } \\
\text { mas asociados } \\
\text { (Factor 3) }\end{array}$ & $\begin{array}{c}\text { Dependencia a los } \\
\text { videojuegos (Factor } \\
\text { general) }\end{array}$ \\
\hline 5 & Muy alto & $6.78 \%$ & $.14 \%$ & $10.43 \%$ & $2.17 \%$ \\
4 & Alto & $18.16 \%$ & $24.80 \%$ & $14.50 \%$ & $22.76 \%$ \\
3 & Esperado & $50.14 \%$ & $50.14 \%$ & $50.14 \%$ & $58.94 \%$ \\
2 & Bajo & $24.93 \%$ & $24.93 \%$ & $22.49 \%$ & $14.36 \%$ \\
1 & Muy bajo & $.00 \%$ & $.00 \%$ & $2.44 \%$ & $1.76 \%$ \\
\hline
\end{tabular}

En la Tabla 7, se observa la prevalencia en cada uno de los niveles de los factores subyacentes al TDV. Lo cual serviría en estudios posteriores que quisieran comparar sus resultados con estas prevalencias.

En la Figura 1, se puede observar que en las barras de error por sexo de cada uno de los factores del TDV, que los hombres con respecto a las mujeres tienden a poseer puntajes significativamente superiores y con una magnitud intermedia en: juego impulsivo $\left(p_{\mathrm{F} 1}=.00 ; d_{\mathrm{F} 1}=.25\right)$, juego compulsivo $\left(p_{\mathrm{F} 2}=.00 ; d_{\mathrm{F} 2}=.72\right)$ y percepción de problemas asociados $\left(p_{\mathrm{F} 3}=.00 ; d_{\mathrm{F} 3}=.48\right)$. Con respecto al
DV, esta diferencia si bien es estadísticamente significativa $\left(p_{\mathrm{DV}}=.03\right)$, es de una magnitud pequeña $\left(d_{\mathrm{DV}}=0.15\right)$.

La distribución de FSJ, revela una distribución marcadamente asimétrica $\left(A s=1.895 ; \sigma_{\mathrm{As}}=.095\right)$, en un rango que abarca las 0 horas, hasta un máximo de 26 horas $(M=3.10$ y $D T=3.55)$. La correlación del TDV con respecto a la FSJ, se hizo con base en el modelo 1.1 (regresión múltiple) y al modelo 1.2 (regresión simple).

En el caso del modelo 1.1 se cumplió el supuesto, $(V I F=1)$, lo que es lógico, ya que las puntuaciones factoriales se calcularon por el método refinado de regresión, 
Tabla 5. Matriz de componentes rotados

\begin{tabular}{|c|c|c|c|c|}
\hline $\begin{array}{l}\mathrm{N}^{\circ} \mathrm{de} \\
\text { ítem }\end{array}$ & Ítem & $\begin{array}{l}\text { Factor 1: Juego } \\
\text { impulsivo }\end{array}$ & $\begin{array}{l}\text { Factor 2: Juego } \\
\text { compulsivo }\end{array}$ & $\begin{array}{l}\text { Factor 3: Percepción de } \\
\text { problemas asociados }\end{array}$ \\
\hline p6 & $\begin{array}{l}\text { Si estoy un tiempo sin jugar me encuentro vacío y no sé qué } \\
\text { hacer }\end{array}$ & .786 & & .312 \\
\hline p8 & $\begin{array}{l}\text { Ya no es suficiente para mí jugar la misma cantidad de tiem- } \\
\text { po que antes, cuando comencé }\end{array}$ & .747 & & \\
\hline p9 & $\begin{array}{l}\text { Dedico menos tiempo a hacer otras actividades, porque los } \\
\text { videojuegos me ocupan bastante rato }\end{array}$ & .740 & & \\
\hline p2 & $\begin{array}{l}\text { Si no me funciona la videoconsola o el PC le pido prestada } \\
\text { una a parientes o amigos }\end{array}$ & .723 & & \\
\hline $\mathrm{p} 5$ & $\begin{array}{l}\text { Dedico mucho tiempo extra con los temas de mis videojue- } \\
\text { gos incluso cuando no estoy jugando con ellos (ver revistas, } \\
\text { hablar con compañeros, dibujar los personajes, etc.) }\end{array}$ & 677 & .343 & \\
\hline $\mathrm{p} 4$ & $\begin{array}{l}\text { Cada vez que me acuerdo de mis videojuegos tengo la nece- } \\
\text { sidad de jugar con ellos }\end{array}$ & 665 & .398 & \\
\hline p11 & $\begin{array}{l}\text { Si no me funciona un videojuego, busco otro rápidamente } \\
\text { para poder jugar }\end{array}$ & 659 & & \\
\hline $\mathrm{p} 10$ & $\begin{array}{l}\text { Estoy obsesionado por subir de nivel, ganar prestigio, etc. en } \\
\text { los videojuegos }\end{array}$ & .643 & .339 & \\
\hline $\mathrm{p} 3$ & $\begin{array}{l}\text { Me afecta mucho cuando quiero jugar y no funciona la vi- } \\
\text { deoconsola o el videojuego }\end{array}$ & .629 & .486 & \\
\hline $\mathrm{p} 13$ & $\begin{array}{l}\text { Me resulta muy difícil parar cuando comienzo a jugar, aun- } \\
\text { que tenga que dejarlo porque me llaman mis padres, amigos } \\
\text { o tengo que ir a algún sitio }\end{array}$ & .614 & .420 & \\
\hline p14 & Cuando me encuentro mal me refugio en mis videojuegos & .607 & & .409 \\
\hline $\mathrm{p} 12$ & Creo que juego demasiado a los videojuegos & .596 & .354 & .353 \\
\hline $\mathrm{p} 1$ & $\begin{array}{l}\text { Juego mucho más tiempo con videojuegos ahora que cuando } \\
\text { comencé }\end{array}$ & .508 & .476 & \\
\hline p18 & Cuando estoy aburrido me pongo con un videojuego & & .784 & \\
\hline p19 & $\begin{array}{l}\text { Me he acostado más tarde o he dormido menos por quedar- } \\
\text { me jugando con videojuegos }\end{array}$ & & .780 & .369 \\
\hline p16 & He llegado a estar jugando más de tres horas seguidas & & .759 & 362 \\
\hline p21 & Cuando estoy jugando pierdo la noción del tiempo & & .745 & \\
\hline $\mathrm{p} 20$ & $\begin{array}{l}\text { En cuanto tengo un poco de tiempo me pongo un videojue- } \\
\text { go, aunque sólo sea un momento }\end{array}$ & & .603 & .427 \\
\hline p7 & $\begin{array}{l}\text { Me irrita cuando no funciona bien el videojuego por culpa } \\
\text { de la videoconsola o el PC }\end{array}$ & .559 & .577 & \\
\hline $\mathrm{p} 15$ & $\begin{array}{l}\text { Lo primero que hago los fines de semana cuando me levanto } \\
\text { es ponerme a jugar con algún videojuego }\end{array}$ & & .528 & .494 \\
\hline p23 & $\begin{array}{l}\text { He mentido a mi familia o a otras personas sobre el tiempo } \\
\text { que he dedicado a jugar }\end{array}$ & & & .742 \\
\hline p24 & $\begin{array}{l}\text { Incluso cuando estoy haciendo otras tareas (en clase, con } \\
\text { mis amigos, estudiando, etc.) pienso en mis videojuegos } \\
\text { (cómo avanzar, superar alguna fase o alguna prueba, etc.) }\end{array}$ & & & .727 \\
\hline p17 & $\begin{array}{l}\text { He discutido con mis padres, familiares o amigos porque de- } \\
\text { dico mucho tiempo a jugar con la videoconsola o el PC }\end{array}$ & & .378 & 690 \\
\hline p22 & $\begin{array}{l}\text { Lo primero que hago cuando llego a casa después de clase o } \\
\text { el trabajo es ponerme con mis videojuegos }\end{array}$ & .304 & .413 & .615 \\
\hline p25 & $\begin{array}{l}\text { Cuando tengo algún problema me pongo a jugar con algún } \\
\text { videojuego para distraerme }\end{array}$ & & .453 & .595 \\
\hline
\end{tabular}

Nota. Análisis de componentes principales. Método de rotación: Varimax con normalización Kaiser. La rotación ha convergido en 6 iteraciones. Solo se presentan las cargas factoriales mayores a 0.30 . 


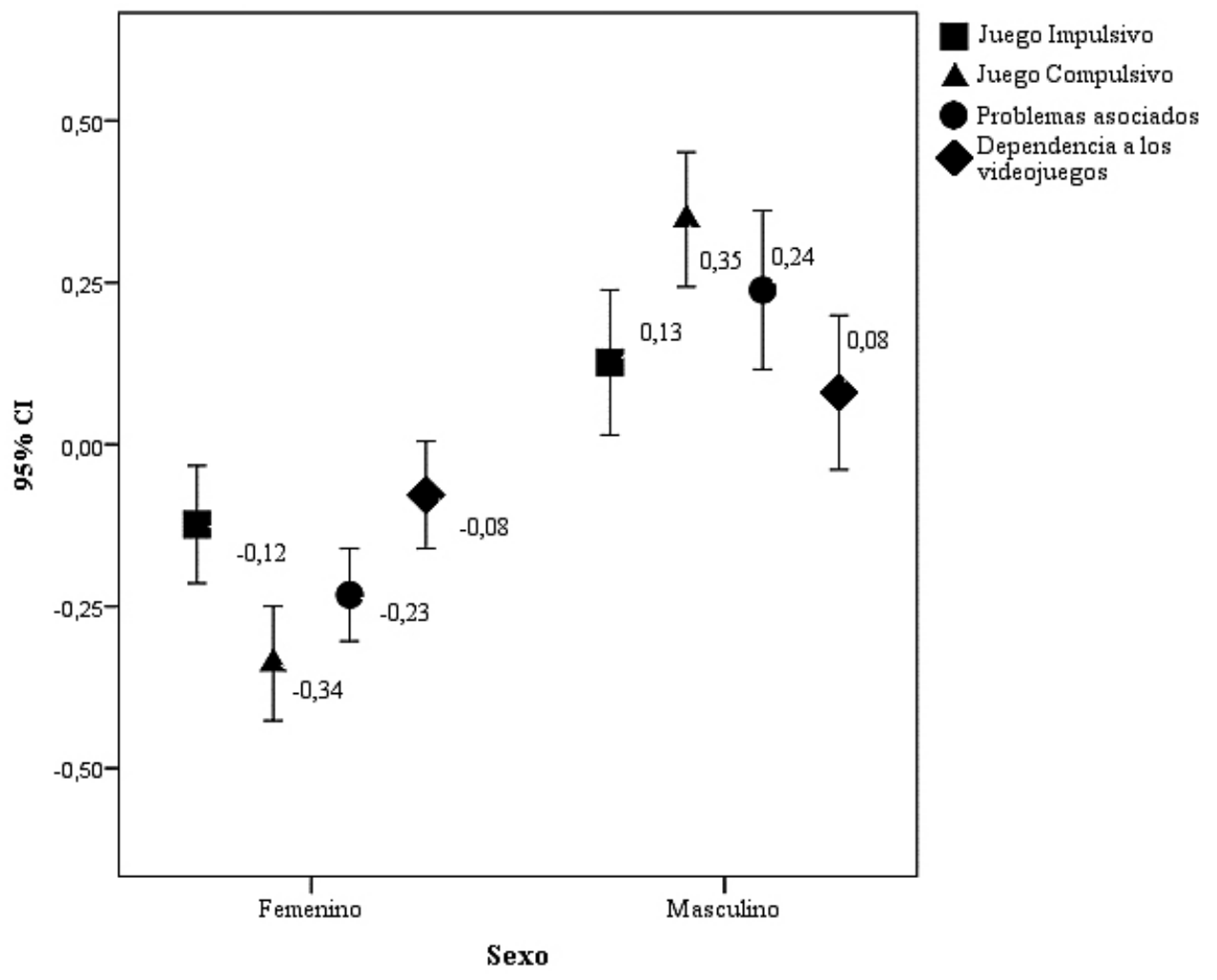

Figura 1. Gráfico de barras de error del TDV por sexo

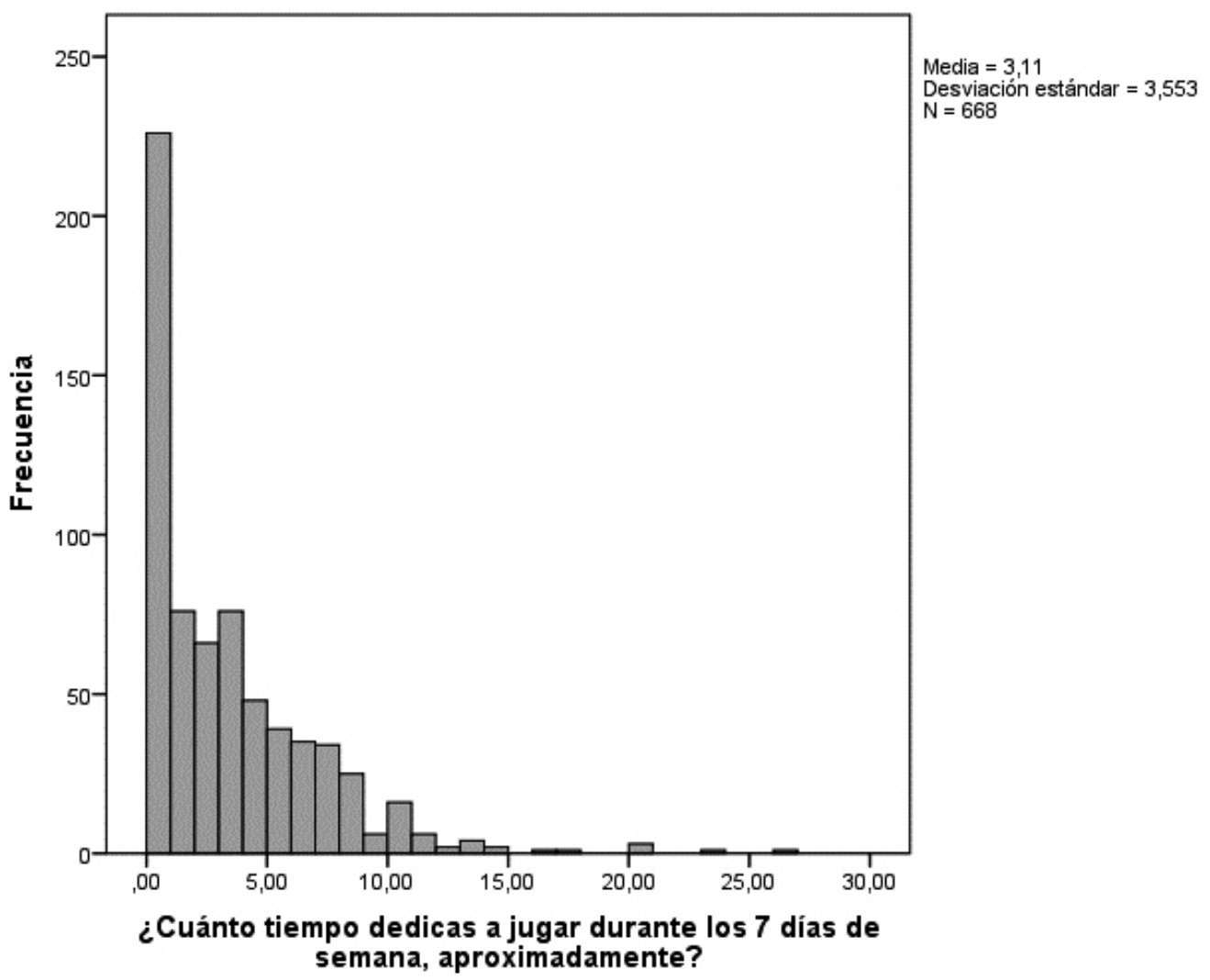

Figura 2. Histograma de la frecuencia (en horas) de juego semanal 
a partir de las cargas factoriales rotadas por Varimax, que asegura que los mismos fuese ortogonales. La relación del modelo 1.1 con la FSJ fue excelente $(R=.701)$ y significativa $(p=.000)$, mientras que el modelo 1.2 fue suficiente $(r=.214)$ y significativa $(p=.000)$, si se interpreta a partir de los criterios descritos por Prieto y Muñiz (2000) o Hernández et al. (2016). Esta diferencia también era esperada, pues el modelo 1.1 tiene más información al tener un mayor número de variables para estimar la FSJ, en comparación con el modelo 1.2. La relación de los factores del TDV con respecto a la FSJ, supone que a mayor puntaje de juego impulsivo $\left(\beta_{\mathrm{F} 1}=\right.$ $\left..187 ; p_{\mathrm{F} 1}=.000\right)$, de juego compulsivo $\left(\beta_{\mathrm{F} 2}=.602 ; p_{\mathrm{F} 2}=\right.$ $.000)$ y de percepción de problemas asociados por el juego $\left(\beta_{\mathrm{F} 3}=.312 ; p_{\mathrm{F} 3}=.000\right)$, mayor será la FJS. La relación del puntaje general de DV, supone que a mayor DV mayor FSJ $\left(\beta_{\mathrm{DV}}=.214 ; p_{\mathrm{DV}}=.000\right)$.

\section{Resumen del modelo}

\begin{tabular}{|l|l|}
\hline Algoritmo & Dos fases \\
\hline Entradas & 3 \\
\hline Agrupaciones & 4 \\
\hline
\end{tabular}

\section{Calidad de clústeres}

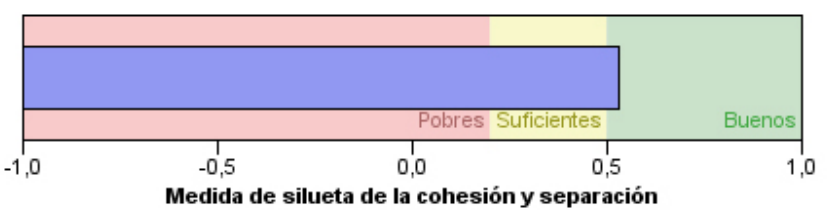

Figura 3. Medida de silueta de la cohesión y separación entre clústeres.

La Figura 3 permite determinar la calidad de la segmentación obtenida. Se encontró que la medida de silueta de la cohesión y separación entre los 4 clústeres obtenidos es buena, al estar por encima del criterio de .50 . Se encontraron 4 clúster, el primero, de mayor tamaño con un $51.4 \%$ de los participantes, el segundo tenía un total del $16.8 \%$ de los participantes, el tercero un $26.3 \%$ de los participantes y finalmente, el cuarto tenía la menor proporción de participantes $(\mathrm{P}=5.6 \%)$. La importancia $(I)$ de los factores del TDV en la construcción de estos clústeres, supone que el juego impulsivo, fue el factor que menos información aportó a su construcción $\left(I_{\mathrm{F} 1}=0.57\right)$, la percepción de problemas asociados fue el segundo factor en importancia $\left(I_{\mathrm{F} 3}=0.99\right) \mathrm{y}$ el juego compulsivo, fue el factor más importante $\left(I_{\mathrm{F} 4}=\right.$ 1.00 ), dado que el valor de su coeficiente $I$ fue el más alto de todos.
Al analizar la Tabla 8 y la Figura 4, se observa que el clúster 1 , aunque es el más grande $(n=349 ; P=$ $51.36 \%$ ), es el más homogéneo en el caso de los factores: juego impulsivo y percepción de problemas asociados, pues el tamaño de las bisagras de estos factores son los más pequeños; igualmente, los parámetros de todos sus factores se encuentran ligeramente por debajo de los parámetros del clúster global, constituyéndose como el clúster más característico de los participantes del estudio, razón por la cual se denominó a este clúster como: tipología de jugador ocasional sin DV. En el caso del clúster 2 , si bien es el tercero más grande $(n=110$; $P=16.47 \%$ ), es el más heterogéneo al tener la mayor amplitud de bisagras en todos los factores del TDV. Con respecto a sus parámetros, este clúster mantiene todas sus bisagras por encima de lo esperado con respecto al clúster global $(M=0.00)$, destacándose por tener un mayor puntaje en el factor de percepción de problemas asociados $(M d=1.58)$, por ello se denominó a este clúster: tipología de jugador problemático con consciencia de DV. En el caso del clúster 3, que es el segundo clúster más grande ( $\mathrm{n}=173 ; P=25.90 \%)$, se observa que es homogéneo a lo interno de todas las bisagras, pero mantiene puntajes elevados, con respecto al parámetro de la distribución general $(M=0.00)$, en el factor de juego compulsivo $(M d=1.16)$ y puntajes bajos en el factor de percepción de problemas asociados $(M d=$ -0.46) por ello se denominó a este clúster: tipología de jugador problemático sin consciencia de DV. Finalmente, el clúster 4 , es el más pequeño ( $n=36 ; P=5.39 \%)$ de todos los clústeres, pero a su vez es el más heterogéneo a lo interno de sus bisagras. Se caracteriza por puntajes altos en el factor de juego impulsivo $(M d=1.07)$ y bajos en los factores de juego compulsivo $(M d=$ $-1.27)$ y percepción de problemas $(M d=-0.70)$, por ello se denominó a este clúster: tipología de jugador frecuente sin DV.

El ANOVA permitió determinar que existen diferencias estadísticamente significativas $(F=111.930 ; p=$ $.000)$ y de gran tamaño $(f=2.05 ; 1-\beta=1.0)$ entre los 4 clústeres. El estadístico de Scheffe (ver Tabla 8), permitió agrupar a los clústers 1 y 4 , por lo que se confirma que ambos serían tipologías sin DV al tener consistentemente $(\alpha=0.05)$ la distribución con menor FJS promedio y los clústers 2 y 3 serían las tipologías con DV al tener consistentemente $(\alpha=0.05)$ la distribución con mayor FJS promedio.

En la Figura 5, se puede observar que los clústeres 1 y 4 se caracterizan por poseer una mayor proporción de mujeres, diferencia que es estadísticamente significativa (Clúster $3_{\mathrm{p}}=.00$; Clúster $\left.4_{\mathrm{p}}=.00\right)$ y con un tamaño del efecto intermedio (Clúster $3_{f}=.37$; Clúster $4_{f}=.21$ ); en 
Tabla 8 Análisis Post - Hoc de Scheffe ${ }^{\mathrm{a}, \mathrm{b}}$ entre los clústeres y la FSJ

\begin{tabular}{lllc}
\hline Clúster & \multirow{2}{*}{\begin{tabular}{c} 
Subconjunto para alfa $[\alpha]=.05$ \\
\cline { 3 - 4 }
\end{tabular}} & & \multicolumn{1}{c}{1} \\
\hline Jugador ocasional sin DV (Clúster 1) & 349 & $52.25 \%$ & 1.32 \\
Jugador frecuente sin DV (Clúster 4) & 36 & $5.39 \%$ & 1.50 \\
Jugador problemático con consciencia de DV (Clúster 2) & 110 & $16.47 \%$ & 5.45 \\
Jugador problemático sin consciencia de DV (Clúster 3) & 173 & $25.90 \%$ & .98 \\
Sig. & & & 5.53 \\
\hline
\end{tabular}

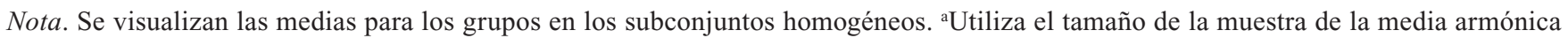

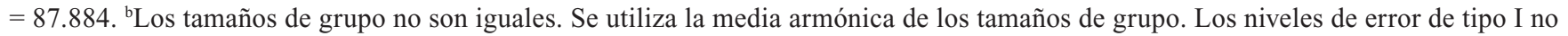
están garantizados.

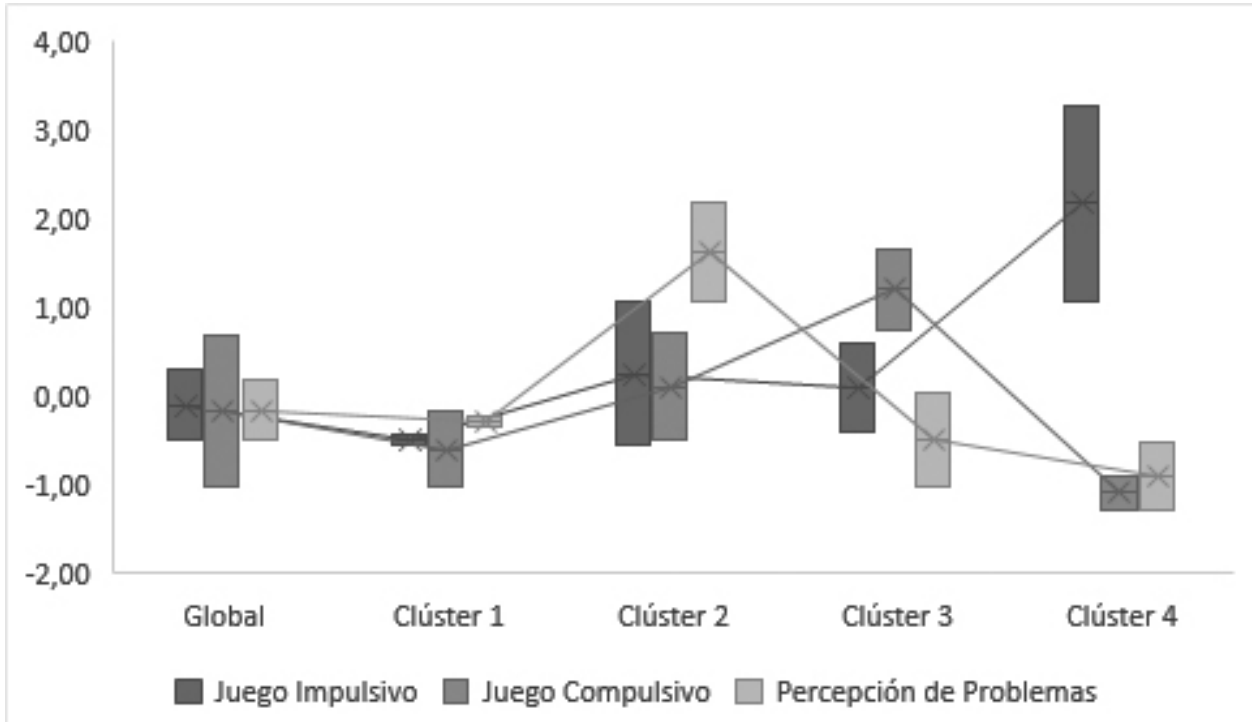

Figura 4. Gráfico de bisagras por factor del TDV a nivel global y por clústeres.

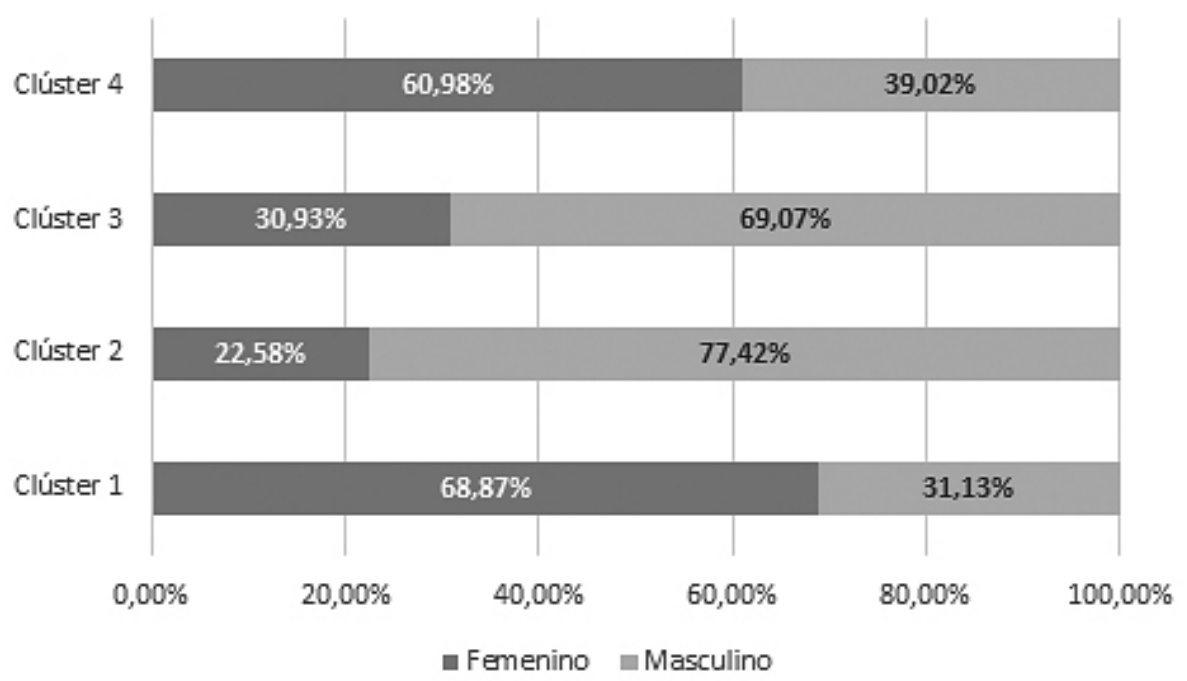

Figura 5. Gráfico de barras apiladas de los clústeres del TDV por sexo. 
cambio, los clústeres 2 y 3 , se caracterizan por poseer una mayor proporción de hombres, diferencia que es es-

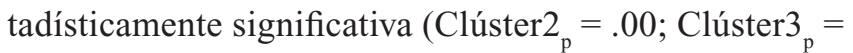
$.00)$ y con un tamaño del efecto intermedio (Clúster $2_{f}=$ .54 Clúster $_{f}=.38$ ).

\section{Discusión}

En el presente estudio se realizó la validación del TDV en Colombia. Se compararon siete modelos factoriales posibles, cinco de los cuales fueron estimados a partir del AFE, coincidiendo uno de ellos (el modelo 3.1 de 1 factor de primer nivel) con los modelos analizados o utilizados en estudios anteriores (Chóliz y Marco, 2011; Salas-Blas et al., 2017) mientras que los restantes dos modelos factoriales verificados (el 5.1 de 4 factores de primer nivel y el 5.2, que es igual que el anterior en el primer nivel, pero agregándole un factor general de segundo nivel) provenían exclusivamente de los modelos planteados por Salas-Blas et al. (2017). Se seleccionaron los modelos 1.1 y 1.2 , caracterizados por tener tres factores de primer nivel (juego impulsivo, juego compulsivo y problemas asociados), diferenciados por el factor de segundo nivel que es la sumatoria ponderada de los factores anteriores cuyo resultado es DV en el modelo 1.2. De ambos modelos, recomendamos el uso del modelo 1.1, al ser un mejor predictor de la FSJ y reproducir mejor las diferencias estadísticamente esperadas por sexo (Wittek et al., 2016), además de permitir la construcción de una tipología, que también reproduce las diferencias por sexo y facilita la comprensdión y la planificación de la intervención clínica por juego problemático (clúster 2 y 3 ).

Nuestros resultados difieren con respecto a los estudios previos (Chóliz y Marco, 2011; Salas-Blas et al., 2017), que señalan que las respuestas al TDV se explican por la presencia de 4 factores de primer nivel y/o de 1 factor subyacente o de segundo nivel. Aunque esto puede atribuirse a diferencias culturales o de edad (considerando que nuestro estudio fue en Colombia y con edades distintas a los estudios hechos en España y Perú), también se debe considerar que los primeros estudios psicométricos del TDV (Chóliz y Marco, 2011; Salas-Blas et al., 2017) no tuvieron en cuenta la minimización de los sesgos de indeterminación factorial, confirmatorio y capitalización del azar, y por tanto, tales diferencias en lo sistemático del tratamiento estadístico podría ser una mejor explicación de las diferencias observadas en las estructuras propuestas en cada estudio, en este caso, el procedimiento utilizado aumentó la validez interna de nuestras conclusiones.

La asimetría positiva de las distribuciones de los factores del TDV y de la FSJ van en línea con los estudios cualitativos de Király y Demetrovics (2017) y de los cuantitativos de Saunders et al. (2017), Van den Brink (2017) y Petry et al. (2015) o Martín et al. (2017), que confirman la baja prevalencia de la $\mathrm{AV}$ en la población general y que puede variar de acuerdo al contexto cultural (Przybylski, 2016). De hecho, Van den Brink (2017), señala que «la prevalencia de problemas de juego varía entre un mínimo del 0.5\% y un máximo del 10\%» (Van den Brink, 2017, p. 291).

Es importante resaltar que los tres factores poseen un valor diferente en el establecimiento de las tipologías y el puntaje total del TDV. El factor 1 (denominado juego impulsivo) es el de menor peso en la predicción de la FSJ y el de menor importancia en el establecimiento de la tipología de DV. Desde un punto de vista clínico, este factor implica «la satisfacción o ejecución del acto impulsivo que estaría estrechamente relacionada con la obtención de un refuerzo positivo» (De Sola et al., 2013, p. 149) y que supondría el estado inicial de la dependencia. Por otro lado, el segundo factor (llamado juego compulsivo) es el de mayor peso al calcular el puntaje total del TDV, además de ser el factor con mayor peso en la predicción de la FSJ, el factor con mayor importancia en el establecimiento de los clústeres y el que posee la diferencia más marcada por sexo a favor de los hombres. Está conformado por diferentes ítems que denotan «la dificultad del control de los impulsos por una sensación subjetiva de incremento de tensión o arousal antes de llevar a cabo el acto impulsivo así como de placer y gratificación tras su realización» (De Sola et al., 2013, p. 149). Al suponer una mayor cronicidad en el control conductual sobre la dependencia, se entiende por qué este factor es el de mayor peso en la relación del TDV con la FSJ y la mayor importancia en el establecimiento de la tipología de DV. Finalmente, el factor 3, el cual hace referencia a la percepción de problemas asociados, implica la apreciación de consecuencias negativas por parte del sujeto a raíz del uso excesivo del videojuego; en especial con la familia, las clases o el trabajo. Este factor, podría ser la vía para que se inicie la búsqueda de tratamiento, pues de este depende el nivel de conciencia que tiene la persona con respecto a los problemas que le generan sus hábitos de juego. Esto podría explicar por qué es el segundo factor con mayor peso con respecto a la predicción de la FSJ y el establecimiento de la tipología de DV. Es importante tener en cuenta que, al momento de calcular el puntaje total del TDV, el factor 3 posee una relación inversa con respecto a los otros dos, lo que indica que este podría ser un factor de protección contra la DV, en la medida en que a mayor puntaje en la percepción de problemas asociados, menor puntaje de DV, lo cual tiene bastante sentido ya que ello implica la presen- 
cia de cierto nivel de autoconciencia con respecto a las consecuencias que tiene el uso en exceso de vídeo juegos.

Los factores encontrados (juegos compulsivos, juego impulsivo y problemas asociados) coinciden con la descripción que hace la CIE-11 (OMS, 2019) con respecto a las características asociadas a la AV. Específicamente, el juego impulsivo (factor 1) se relacionaría con la falta de control de la conducta de juego en cuanto al inicio, frecuencia, intensidad, duración, finalización y contexto en que se juega; el juego compulsivo (factor 2) con el aumento de la prioridad que se otorga a los juegos frente a otros intereses vitales y actividades diarias y, finalmente, la percepción de problemas asociados (factor 3) con el mantenimiento o escalada de la conducta de juego a pesar de tener conciencia de las consecuencias negativas.

Las limitaciones de este estudio se relacionan con la estrategia de muestreo utilizada debido a que los parámetros poblacionales de Colombia no fueron equivalentes a los de los estudios previos de Chóliz y Marco (2011) o de Salas-Blas et al. (2017), es decir, los resultados del presente trabajo no se pueden generalizar a la población de los estudios originales. Por ésta razón, se sugiere maximizar la validez externa de nuestro modelo factorial explicativo de las respuestas del TDV a partir de investigaciones que se centren en el análisis de su invarianza factorial en otras poblaciones, incluyendo las ya estudiadas por Chóliz y Marco (2011) y Salas-Blas et al (2017).

En aras de construir una mejor teoría sobre la $\mathrm{AV}$, se sugiere realizar estudios longitudinales (Lopez et al., 2017) para profundizar en la etiología del trastorno, especialmente por el tránsito que una persona puede tener entre cada una de las tipologías encontradas, ya que si consideramos la relación euforia - disforia que sucede en las adicciones comportamentales (de Sola et al., 2013), podríamos esperar que exista un orden temporal entre ellas, al pasar de un vínculo normal (Clúster 1), al establecimiento del hábito de juego por el placer que ésta actividad genera (Clúster 4) y hasta que, como consecuencia de la abstinencia que se genera por no jugar, se incrementa exageradamente el hábito de juego (Clúster 3 ); y finalmente, la persona adquiere consciencia del problema (Clúster 2) generando un «estado de stress o ansiedad [...], así como una disminución del estado disfórico una vez llevado a cabo el comportamiento [de juego..., que] se encuentra relacionada con mecanismos de refuerzo negativo y de conductas automáticas para su consecución» (de Sola et al., 2013, p149). Así mismo, el uso de las tipologías para caracterizar a los individuos refleja también las diferencias estadísticamente esperadas por sexo (Wittek et al., 2016) y por FSJ, dado a que en los clústeres 2 y 3 , es donde se observa una mayor propor- ción de hombres, además de ser los clústeres en donde hubo una mayor FSJ, por lo que creemos que es donde se manifiesta un mayor juego problemático o de DV.

En conclusión, se realizó un análisis riguroso y sistemático, de las propiedades psicométricas del TDV, con miras a validarlo en Colombia, implementando mejoras con respecto a estudios de validación realizados anteriormente, y con miras a cubrir la necesidad de poder utilizar un test adecuado para evaluar el grado de adicción a los videojuegos o de juego problemático en nuestro contexto. Adicional a esto, se estableció una tipología de los jugadores con base en estos factores, y se presentaron normas específicas de corrección del instrumento, para que puedan ser de utilidad práctica para quienes lo realicen. Se espera que el TDV pueda ser utilizado tanto por psicólogos clínicos, psiquiatras, o distintos profesionales de la salud, así como que sea de utilidad para propósitos investigativos.

\section{Conflictos de interés}

Los autores declaran no existen conflictos de intereses

\section{Referencias}

Aarseth, E., Bean, A., Boonen, H., Colder, M., Coulson, M., Das, D., \& Van Rooij, A. (2016). Scholars' open debate paper on the World Health Organization ICD-11 Gaming Disorder proposal. Journal of Behavioral Addictions, 6(3), 1-4. https:// doi.org/10.1556/2006.5.2016.088

Asociación Americana de Psiquiatría. (2013) Manual de diagnóstico y estadística de trastornos mentales ( $5^{\mathrm{a}}$ ed.). Autor.

Batista, J. y Coenders, G. (2000). Modelos de ecuaciones estructurales: modelos para el análisis de relaciones causales. La muralla.

Briones, G. (1996). Metodología de la Investigación Cuantitativa en Ciencias Sociales. Instituto Colombiano para el Fomento de la Educación Superior.

Buiza-Aguado, C., García-Calero, A., Alonso-Cánovas, A., OrtizSoto, P., Guerrero-Díaz, M., González-Moliniere, M. y Hernández-Medrano, I. (2017). Los videojuegos: una afición con implicaciones neuropsiquiátricas. Psicología Educativa, 23(2), 129-136. https://doi.org/10.1016/j.pse.2017.05.001

Chóliz, M. y Marco, C. (2011). Patrón de uso y dependencia de videojuegos en infancia y adolescencia. Anales de Psicología 27(2), 418-426. https://revistas.um.es/analesps/article/ view/123051

Departamento Administrativo Nacional de Estadística (8 de noviembre de 2018). Proyecciones y retroproyecciones de población calculadas con base en los resultados del Censo Nacional de Población y Vivienda-CNPV-2018. Sitio web del Departamento Administrativo Nacional de Estadística: https:// www.dane.gov.co/index.php/estadisticas-por-tema/ demografia-y-poblacion/proyecciones-de-poblacion. 
De Sola, J., Rubio, G. y Rodríguez, F. (2013). La impulsividad: ¿antesala de las adicciones comportamentales? Salud y Drogas, 13(2),145-155.https://www.redalyc.org/pdf/839/83929573007. pdf

DiStefano, C., Zhu, M., \& Mîndrilă, D. (2009). Understanding and using factor scores: Considerations for the applied researcher. Practical Assessment, Research \& Evaluation 14 (20), 1-11. https://doi.org/10.7275/da8t-4g52

Dunn, T., Baguley, T., \& Brunsden, V. (2013). From alpha to omega: A practical solution to the pervasive problem of internal consistency estimation. British Journal of Psychology, 105(3), 399-412. https://doi.org/10.1111/bjop.12046

Eiroa, F., Fernandez, I., \& Perez, P. (2008). Psychological questionnaires and research on the Internet: A literature review. Anales de Psicologia, 24(1), 150-157. https://revistas. um.es/analesps/article/view/32871

Freiberg, A., Stover, J., De la Iglesia, G. y Fernandez, M. (2013). Correlaciones policóricas y tetracóricas en estudios factoriales exploratorios y confirmatorios. Ciencias Psicológicas, 12,1 51-164. http://www.scielo.edu.uy/scielo.php?script=sci arttextypid $=\mathrm{S} 1688-42212013000200005 \mathrm{ylng}=\mathrm{esytlng}=\mathrm{en}$.

Hair, J., Black, W., Babin, B., \& Anderson, R. (2014). Multivariate Data Analysis ( $7^{\text {th }}$ ed.,). Pearson Education.

Hernández, A., Ponsoda, V., Muñiz, J., Prieto, G. y Elosua, P. (2016). Revisión del modelo para evaluar la calidad de los tests utilizados en España. Papeles del Psicólogo, 37(3), 192-197. https://www.redalyc.org/articulo.oa?id=778/77847916006

King, D., Haagsma, M., Delfabbro, P., Gradisar, M., \& Griffiths, M. (2013). Toward a consensus definition of pathological video-gaming: A systematic review of psychometric assessment tools. Clinical Psychology Review, 33(3), 331342. https://doi.org/10.1016/j.cpr.2013.01.002

Király, O. \& Demetrovics, Z. (2017). Inclusion of Gaming Disorder in ICD has more advantages than disadvantages Commentary on: Scholars' open debate paper on the World Health Organization ICD-11 Gaming Disorder proposal (Aarseth et al.). Journal of Behavioral Addictions, 6 (3), 280284. https://doi.org/10.1556/2006.6.2017.046

Király, O., Sleczka, P., Pontes, H., Urbán, R., Griffiths, M., \& Demetrovics, Z. (2017). Validation of the Ten-Item Internet Gaming Disorder Test (IGDT-10) and evaluation of the nine DSM-5 Internet Gaming Disorder criteria. Addictive Behaviors, 64, 253-260. https://doi.org/10.1016/j.addbeh.2015.11.005

Ko, C., Yen, J., Chen, S., Wang, P., Chen, C., \& Yen, C. (2014). Evaluation of the diagnostic criteria of Internet gaming disorder in the DSM-5 among young adults in Taiwan. Journal of Psychiatric Research, 53, 103-110. https://doi. org/10.1016/j.jpsychires.2014.02.008

Kolenikov, S. \& Bollen, K. (2012). Testing Negative Error Variances: Is a Heywood Case a Symptom of Misspecification? Sociological Methods y Research, 41(1), 124-167. https://doi. org/10.1177/0049124112442138

Lloret, S., Ferreres, A., Hernández, A. y Tomás, I. (2014). El análisis factorial exploratorio de los ítems: una guía práctica, revisada y actualizada. Anales de Psicología, 30 (3), 11511169. https://doi.org/10.6018/analesps.30.3.199361

Lemmens, J., Valkenburg, P., \& Gentile, D. (2015). The Internet Gaming Disorder Scale. Psychological Assessment, 27(2), 567-582. https://doi.org/10.1037/pas0000062
Lopez-Fernandez, O., Kuss, D., Pontes, H., \& Griffiths, M. (2016). Video game addiction: Providing evidence for Internet gaming disorder through a systematic review of clinical studies. European Psychiatry, 33, S306. https://doi.org/10.1016/j. eurpsy.2016.01.1047

Lorenzo-Seva, U. \& Ferrando, P. (2013). Factor 9.2: A comprehensive program for fitting exploratory and semiconfirmatory factor analysis and IRT models. Applied Psychological Measurement, 37(6), 497-498. https://doi. org/10.1177/0146621613487794

Marengo, L., Herrera, L., Coutinho, T., Rotela, G., \& Strahler, T. (2015). Gamer o adicto? Revisión narrativa de los aspectos psicológicos de la adicción a los videojuegos. Revista Neuropsicologia Latinoamericana, 7(3), 1-12. https://doi. org/10.5579/rnl.2015.0266

Martín, M., Matalí, J., García, S., Pardo, M., Lleras, M., y Castellano, C. (2017). Adolescentes con Trastorno por juego en Internet (IGD): perfiles y respuesta al tratamiento. Adicciones, 29(2), 125-133. https://doi.org/10.20882/adicciones.890

McDonald, R. (1999). Test theory: a unified treatment. Lawrence Earlbaum Associates. Inc.

Mentzoni, R., Brunborg, G., Molde, H., Myrseth, H., Skouverøe, K., Hetland, J., \& Pallesen, S. (2011). Problematic video game use: Estimated prevalence and associations with mental and physical health. Cyberpsychology, Behavior, and Social Networking, 14(10), 591-596. https://doi.org/10.1089/cyber.2010.0260

Müller, K. (2017). Under the umbrella. Commentary on: Chaos and confusion in DSM-5 diagnosis of Internet Gaming Disorder: Issues, concerns, and recommendations for clarity in the field (Kuss et al.). Journal of Behavioral Addictions, 6(3), 313-316. https://doi.org/10.1556/2006.6.2017.011

Organización Mundial de la Salud (2019). CIE-11 para estadísticas de mortalidad y morbilidad (Versión: 04 / 2019). https://icd.who.int/browse11/1-m/es

Petry, N., Rehbein, F., Ko, C., \& O’Brien, C. (2015). Internet Gaming Disorder in the DSM-5. Current Psychiatry Reports, 17 (1), 1-9. https://doi.org/10.1007/s11920-015-0610-0

Pinzon, N. y Briñez, L. (2018). Propiedades psicométricas del Cuestionario de Adicción a Internet y a los Videojuegos para Adolescentes. Universitas Psychologica, 17(4), 1-13. https:// doi.org/10.11144/Javeriana.upsy17-4.ppca

Pontes, H. \& Griffiths, M. (2014). Assessment of Internet gaming disorder in clinical research: Past and present perspectives. Clinical Research and Regulatory Affairs, 31(2-4), 35-48. https://doi.org/10.3109/10601333.2014.962748

Prieto, G. y Muñiz, J. (2000). Un modelo para evaluar la calidad de los tests utilizados en España. Papeles del psicólogo, 77, 65-72. https://www.redalyc.org/articulo.oa?id=778/77807709

Przybylski, A. (2016). Mischievous responding in Internet Gaming Disorder research. PeerJ, 4(e2401). https://doi. org/10.7717/peerj.2401

Salas-Blas, E., Merino-Soto, C., Chóliz, M., y Marco, C. (2017). Análisis psicométrico del test de dependencia de videojuegos (TDV) en población peruana. Universitas Psychologica, 16(4), 1-13. https://doi.org/10.11144/Javeriana.upsy16-4.aptd

Saunders, J., Hao, W., Long, J., King, D., Mann, K., Fauth-Bühler, M., Rumpf, H., Bowden-Jones, H., Rahimi-Movaghar, A., Chung, T., Chan, E., Bahar, N., Achab, S., Lee, H., Potenza, M., Petry, N., Spritzer, D., Ambekar, A., Derevensky, J., ... 
Poznyak, V. (2017). Gaming disorder: Its delineation as an important condition for diagnosis, management, and prevention, Journal of Behavioral Addictions, 6(3), 271-279. https://doi.org/10.1556/2006.6.2017.039

Tejeiro, R. \& Bersabé, M. (2002). Measuring problem video game playing in adolescents. Addiction, 97 (1), 1601-1606. https:// doi.org/10.1046/j.1360-0443.2002.00218.x

Tukey, J. (1976). Exploratory Data Analysis. Addison-Wesley Van den Brink, W. (2017). ICD-11 Gaming Disorder: Needed and just in time or dangerous and much too early? Journal of Behavioral Addictions, 6 (3), 290-292. https://doi. org/10.1556/2006.6.2017.040

Van Rooij, A., Schoenmakers, T., \& Van de Mheen, D. (2017). Clinical validation of the C-VAT 2.0 assessment tool for gaming disorder: A sensitivity analysis of the proposed DSM5 criteria and the clinical characteristics of young patients with 'video game addiction. Addictive Behaviors, 64 (1), 269274. https://doi.org/10.1016/j.addbeh.2015.10.018
Van Rooij, A., Schoenmakers, T., Van den Eijnden, R., Vermulst, A., \& Van de Mheen, D. (2012). Video game addiction test: validity and psychometric characteristics. Cyberpsychology, Behavior, and Social Networking, 15(9), 507-511. https://doi. org/10.1089/cyber.2012.0007

Walther, B., Morgenstern M. \& Hanewinkel R. (2012). CoOccurrence of Addictive Behaviours: Personality Factors Related to Substance Use, Gambling and Computer Gaming. European Addiction Research, 18 (1), 167-174. https://doi. org/10.1159/000335662

Wittek, C., Finserås, T., Pallesen, S., Mentzoni, R., Hanss, D., Griffiths, M., \& Molde, H. (2016). Prevalence and Predictors of Video Game Addiction: A Study Based on a National Representative Sample of Gamers. International Journal of Mental Health and Addiction, 14(5), 672-686. https://doi. org/10.1007/s11469-015-9592-8

Young, K. (2 de septiembre de 2018). Video and online game addiction test. Net Addiction. Sitio web de Netadicction: http://netaddiction.com/are-you-an-obsessive-online-gamer/ 